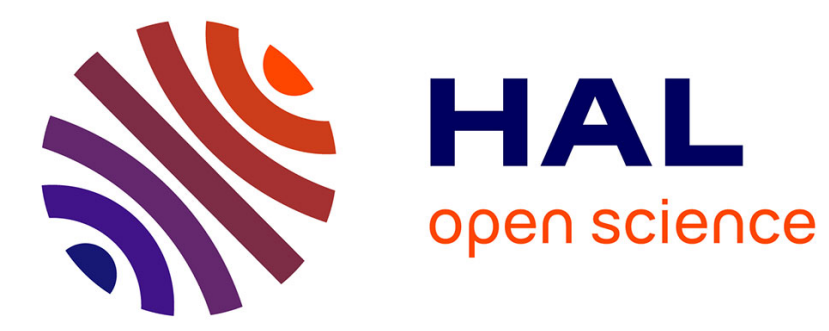

\title{
A Delay Probability Metric for Input Pattern Ranking Under Process Variation and Supply Noise
}

Anu Asokan, Aida Todri-Sanial, Alberto Bosio, Luigi Dilillo, Patrick Girard, Serge Pravossoudovitch, Arnaud Virazel

\section{- To cite this version:}

Anu Asokan, Aida Todri-Sanial, Alberto Bosio, Luigi Dilillo, Patrick Girard, et al.. A Delay Probability Metric for Input Pattern Ranking Under Process Variation and Supply Noise. ISVLSI: International Symposium on Very Large Scale Integration, Jul 2014, Tampa, FL, United States. pp.226-231, 10.1109/ISVLSI.2014.42 . lirmm-01248592

\section{HAL Id: lirmm-01248592 \\ https://hal-lirmm.ccsd.cnrs.fr/lirmm-01248592}

Submitted on 17 Jul 2019

HAL is a multi-disciplinary open access archive for the deposit and dissemination of scientific research documents, whether they are published or not. The documents may come from teaching and research institutions in France or abroad, or from public or private research centers.
L'archive ouverte pluridisciplinaire HAL, est destinée au dépôt et à la diffusion de documents scientifiques de niveau recherche, publiés ou non, émanant des établissements d'enseignement et de recherche français ou étrangers, des laboratoires publics ou privés. 


\title{
A Delay Probability Metric for Input Pattern Ranking Under Process Variation and Supply Noise
}

\author{
Anu Asokan, Aida Todri-Sanial, Alberto Bosio, Luigi Dilillo, Patrick Girard, \\ Serge Pravossoudovitch, Arnaud Virazel \\ LIRMM-University of Montpellier II/CNRS, Montpellier, France \\ Email: lastname@lirmm.fr
}

\begin{abstract}
Ongoing technology scaling has increased delay defects in integrated circuits. Some of the delay defects are due to crosstalk, supply noise, process variations, etc. They degrade the performance and field reliability of circuits. However, testing the circuits with path delay patterns under worst-case conditions helps to detect such defects. Estimation of patterns with worst-case path delay becomes difficult using the conventional techniques due to their unpredictable behavior. In this paper, we first describe the problem and then propose our approach in identifying a worst-case path delay pattern under the impact of process variations and supply noise. A delay probability metric is presented in this work, for an efficient identification of worst-case path delay pattern, which is the basis of our ranking method. The simulation results of ITC'99 benchmark circuits show the feasibility of our delay probability metric.
\end{abstract}

Index Terms-delay defects, delay probability metric, process variations $(\mathrm{PV})$, supply noise $(\mathrm{SN})$

\section{INTRODUCTION}

Ongoing technology scaling has increased delay defects in integrated circuits. Their early detection, lowers defect escape rate and ensures better circuit performance and field reliability. Delay defects are majorly introduced by the variations in the manufacturing process, as well as the noise disturbances in the power supply and ground networks. Timing-aware automatic test pattern generation (ATPG) tools are commercially utilized to detect delay-related defects. But they are incapable of accurately generating the worst-case path delay pattern as they do not consider physical defects affecting the gates, interconnects, power supply and ground networks, etc. Gate or path delay can be modeled using statistical static timing analysis (SSTA) [1]. SSTA and process corner based techniques work with delay probability distributions. However, they are too complex to work with realistic path delay distributions and identify an accurate delay pattern. This has motivated us to propose a simple and novel delay probability metric to identify a worstcase path delay pattern for capturing delay defects.

Unpredictable process parameter fluctuations and changing operating voltage conditions cause random variations in the circuit parameters, thereby affecting the expected nominal path delay values. Fluctuations in the manufacturing process affect the gate parameters such as threshold voltage $\left(V_{t h}\right)$, oxide thickness $\left(t_{o x}\right)$, transistor length $\left(L_{g}\right)$ and width $\left(W_{g}\right)$, as well as the width of interconnects (resistance, inductance and capacitance). Operating voltage of a circuit varies depending on the noise disturbances in their power supply (i.e., power supply noise) or ground networks (i.e., ground bounce), varying the drive strength in the gates. Also, path delay of a circuit varies randomly depending on the input patterns and their arrival times. The combined impact of all these effects makes path delay estimation difficult. Therefore, a simple yet effective method for identifying the worst-case or the most effective path delay patterns that can capture a delay defect during testing is essential. In this work, we propose a delay probability metric to identify a worst-case path delay pattern in the presence of $\mathrm{PV}$ and $\mathrm{SN}$.

There are a number of contributions that investigate the impact of PV and SN. Based on the source of physical defects, they can be classified as delay defect: (1) due to a single source (i.e., PV, SN or crosstalk only), (2) from multiple sources, and (3) irrespective of the source. The first classification focuses only on process variations [2]-[5]. Francisco et al. [2] proposed a statistical timing analysis framework based on delay correlation information between two paths. Critical path delay measurement using a ring oscillator is presented in [3]. Authors in [4] have proposed an algorithm to detect a resistive interconnect defect for a path with minimum delay variance. An optimization framework is suggested by $\mathrm{Yu}$ [5] based on SSTA for worst-case circuit analysis. In the second classification, different approaches for pattern generation from multiple sources were proposed [6]-[8]. Todri et al. [6] has analyzed power supply noise and ground bounce for capturing worst-case path delay patterns based on simulated annealing. $\mathrm{Xu}$ [7] described a statistical model for skitter with PV and power supply noise effects. Peng [8] explains their work of pattern evaluation and selection considering crosstalk and PV. In the third classification, worst-case delay of a circuit path is analyzed, with no detailed reference to the source of defects. A theoretical framework for statistical timing analysis is proposed by Orshansky and Keutzer [9] for detecting the worst-case path delay in a circuit.

In contrast to all these works, our goal is to re-examine the problem of path delay pattern generation by introducing a delay probability metric for ranking patterns under the impact of PV and SN. Using the probabilistic metrics, we can estimate and identify an efficient worst-case path delay pattern or set of patterns that can capture the worst path delay. Our method is practical and easily adaptable to be implemented on any existing pattern generation flow. Complementary to the previous works, we have additionally incorporated the impact 
of ground bounce in supply noise. Our major contributions in this paper are summarized as follows:

- A probability metric is presented to identify worst-case path delay pattern while considering the combined impact of PV and SN. This metric aims at detecting the most-effective pattern for path delay testing from the subset of all input patterns.

- Ranking method is described based on the mean delay difference and the area of the delay probability distribution of all input patterns.

- Case study and simulation results are shown to validate our method.

The rest of this paper is organized as follows. In Section II, we formulate the problem of path delay distribution for detecting a worst-case pattern in the presence of PV and SN. Section III demonstrates the individual and combined impact of PV and SN. Section IV presents the simulation results on ITC'99 benchmark circuits. Finally, in Section V, we conclude our paper.

\section{PROBLEM FORMULATION}

In this section, we describe the problem that we address and propose our mathematical approach in identifying test patterns that can capture the worst-case path delay under the impact of PV and SN. Initially, we estimate the dependent circuit parameters that affect the delay of a circuit path. Then, describe the proposed probabilistic metrics for ranking patterns based on their effectiveness. Finally, we describe our method of ranking patterns based on a delay probability metric.

\section{A. Path Delay Estimation}

In this subsection, we describe the proposed analytical method for computing the ranking order of test patterns. The problem of path delay test is a well-understood and widely investigated problem by the scientific community, in effectively identifying the test patterns for performance evaluation that would eventually lead to high-quality test patterns that can lower defect escape rate. With technology scaling, increased circuit densities and faster switching circuits, identifying the high quality patterns is getting even more challenging. Even more so when the impact of physical design issues and PV are taken into account. Due to the nature of the problem with many parameters that can cause a wide delay distribution, we exploit a probability based-approach to rank patterns based on their effectiveness for capturing the worst case delay under the impact of PV and SN.

Each test pattern triggers a given switching activity on the circuit and the critical path under observation. As already shown in [6], critical paths can undergo drastic delay variation that can lead to slow-down and/or speed-up effects. We expect that such delay variations will be even more pronounced when PV of transistors and interconnects are included.

Problem definition: We aim to identify the set of patterns that are the most effective for capturing the worst case path delay under the impact of PV and $\mathrm{SN}$; based on the delay probability density function of each pattern.
Path delay on a circuit is computed by considering the delays of both interconnects and gates. Supply noise which exhibits itself as power and ground voltage fluctuations can impact the operating regions of transistors, hence the delay behavior of the gates. Additionally, different gates on a path can suffer from different amounts of supply noise. Random process variations induce deviation on the transistor's and interconnect's dimensions and carrier's mobility. In this work, we consider process variation on threshold voltage, $V_{t h}$, oxide thickness, $t_{o x}$, transistor gate length, $L_{g}$ and width, $W_{g}$ and interconnect length and width that impact interconnect parasitics, $R, L, C$. From supply noise perspective, we consider noise on power, $V_{d d}$ and ground, $G_{n d}$.

The delay of a pMOS transistor due to a rising input can be expressed as [10]:

$$
\begin{array}{r}
\delta_{r}=\frac{2 C_{L}}{\beta_{p}\left[\left(V_{d d}-G_{n d}\right)-\left|V_{t h p}\right|\right]}\left[\frac{\left|V_{t h p}\right|-0.1\left(V_{d d}-G_{n d}\right)}{\left[\left(V_{d d}-G_{n d}\right)-\left|V_{t h p}\right|\right]}\right. \\
\left.+\frac{1}{2} \log \left|\frac{19\left(V_{d d}-G_{n d}\right)-20\left|V_{t h p}\right|}{\left(V_{d d}-G_{n d}\right)}\right|\right]
\end{array}
$$

where $C_{L}$ is the load capacitance including the next stage load and interconnect capacitance and $V_{t h p}$ is the threshold voltage of pMOS transistor. Similarly, the transistor delay for a falling input can be expressed as [10]:

$$
\begin{array}{r}
\delta_{f}=\frac{2 C_{L}}{\beta_{n}\left[\left(V_{d d}-G_{n d}\right)-V_{t h n}\right]}\left[\frac{V_{t h n}-0.1\left(V_{d d}-G_{n d}\right)}{\left[\left(V_{d d}-G_{n d}\right)-V_{t h n}\right]}\right. \\
\left.+\frac{1}{2} \log \left|\frac{19\left(V_{d d}-G_{n d}\right)-20 V_{t h n}}{\left(V_{d d}-G_{n d}\right)}\right|\right]
\end{array}
$$

where $V_{t h n}$ is the threshold voltage of nMOS transistor and $\beta$ is the transistor gain factor (in pMOS and nMOS), can be expressed as:

$$
\beta=\frac{\mu \epsilon}{t_{o x}}\left(\frac{W_{g}}{L_{g}}\right)
$$

where $\mu$ is the effective surface mobility of the carriers in the channel, and $\epsilon$ is the permittivity of the gate insulator. Based on the transistor delay, the gate delay can be computed, such as for an inverter the average gate delay can be computed as:

$$
\delta_{g}=\left(\delta_{f}+\delta_{r}\right) / 2
$$

Interconnects are usually modeled as $\pi$-networks with $R L C$ parasitics, their delay, $\delta_{\text {int }}$ can be computed by applying Elmore delay formulation as a function of $\zeta_{i}$ at node $i$, as in [11]:

$$
\delta_{i n t}=1.047 e^{\frac{-\zeta_{i}}{0.85}}+1.39 \zeta_{i}
$$

where $\zeta_{i}$ is expressed as:

$$
\zeta_{i}=\frac{1}{2}\left(\frac{\sum_{k} C_{k} R_{i k}}{\sqrt{\sum_{k} C_{k} L_{i k}}}\right)
$$


where $R_{k}$ is the interconnect resistance, $C_{k}$ is the interconnect capacitance, $L_{k}$ is the interconnect inductance and $k$ represents the number of elements on the $\pi$-network interconnect model. Hence, the delay on a path can be computed as the sum of gate delays and interconnects delays (i.e. for $n$ gates and $n-1$ interconnects on a given path) that are triggered by a given input pattern as:

$$
\delta_{\text {path }}=\sum_{i=1}^{n} \delta_{g_{i}}+\sum_{i=1}^{n-1} \delta_{i n t_{i}}
$$

\section{B. Delay Probability Distribution}

For a given path, the delay would be a function of many variables due to PV and SN. Path delay variations due to these variables can be expressed as a function of parameters as:

$$
\begin{array}{r}
\delta_{\text {path }}=f\left(V_{d d}, G_{n d}, V_{t h}, t_{o x}, L_{g}, W_{g}, R, L, C, C_{L}\right) \\
=f(S N, P V)
\end{array}
$$

Definition: In general terms, the path delay variation, $\delta_{\text {path }}$ for a given input pattern, $P I$ can be represented as a normal distribution function. As path delay (due to $\delta_{g_{i}}$ or $\delta_{i n t_{i}}$ ) can be real-valued random values whose distribution are unknown, the highest probability of worst-case path delay can be observed better using a normal delay distribution function. The path delay due to test patterns for all parameters (PV and $\mathrm{SN}$ ) can be expressed as normal distribution $N\left(\mu_{P I}, \sigma_{P I}\right)$.

The mean and standard deviation of a path delay for a given input pattern, $P I$ and all parameters can be expressed as:

$$
\begin{gathered}
\mu_{P I}=\frac{1}{N} \sum_{i=1}^{N} \delta_{\text {path }_{i}} \\
\sigma_{P I}=\sqrt{\frac{1}{N} \sum_{i=1}^{N}\left(\delta_{\text {path }_{i}}-\mu_{P I}\right)^{2}}
\end{gathered}
$$

where $N$ represents the total number of path delay measurements for a given path under PV and SN parameters.

\section{Probabilistic Pattern Ranking Method}

Here, we describe the concept of deriving pattern ranking method utilizing the path delay distribution function. Fig. 1 illustrates the probability density distribution of an input pattern under process variation and supply noise. Assuming that for a known design, there is a predefined delay threshold with $\mu_{\text {nom }}$ that represents the tolerable delay of the circuit.

Definition: We define the probability of identification, $P_{\text {identification }}$ that can analytically estimate the likeliness of a pattern $j$ under PV and SN conditions to cause a path delay at each node $i, \delta_{\text {path }_{i}}$ larger than the allowed delay threshold, $\mu_{\text {nom }}$, and can be expressed as:

$$
P_{\text {identification }_{j}}\left[\mu_{P I_{j}} \geq \mu_{\text {nom }}\right]=\int_{\mu_{\text {nom }}}^{\mu_{\text {max }_{j}}} \delta_{\text {path }_{i}}(t) \partial t
$$

where $\mu_{\max _{j}}$ of a pattern $j$ is defined as:

$$
\mu_{\max _{j}}=\mu_{P I_{j}}+\frac{3 \sigma_{P I_{j}}}{2}
$$

Hence, for each pattern, the $P_{\text {identification }_{j}}$ allows us to compute the exposed area of the probability density function beyond a delay threshold also as shown in Fig.1. Utilizing this metric, we further define the pattern ranking method that considers both the mean, $\mu_{P I_{j}}$ and probability of identification, $P_{\text {identification }_{j}}$ of each pattern for classifying the patterns for inducing the worst path delay under PV and SN conditions. The ranking metric, $\operatorname{Rank}_{P I_{j}}$ is defined as:

$$
\operatorname{Rank}_{P I_{j}}=\alpha_{1} \mu_{P I_{d}}+\alpha_{2} P_{\text {identification }_{j}}
$$

where $\alpha_{1}$ and $\alpha_{2}$ are weight coefficients between 0 to 1 that can be given for taking into account both the changes in mean, $\mu_{P I_{d}}$ and the identification metric $P_{\text {identification }_{j}}$, where $\mu_{P I_{d}}$ is expressed as:

$$
\mu_{P I_{d}}=\mu_{n o m}-\mu_{P I_{j}}
$$

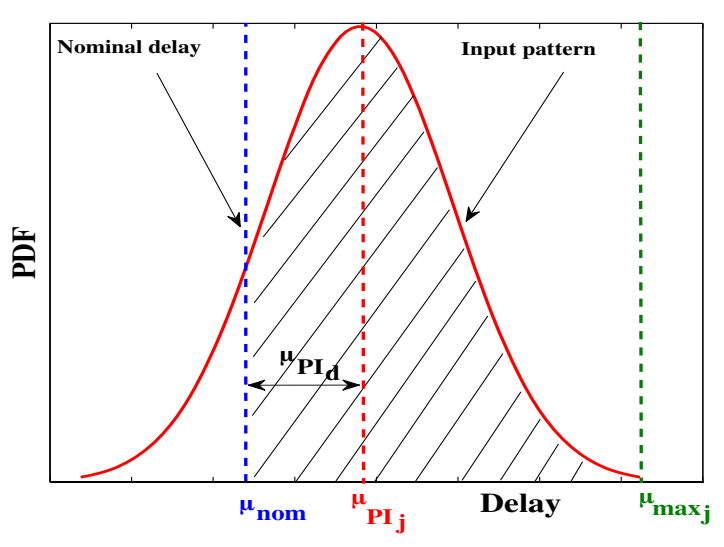

Fig. 1. Delay Probability distribution of an input pattern

The values for $\alpha_{1}$ and $\alpha_{2}$ can be chosen based on their priority (i.e., $\mu_{P I_{d}}$ or $P_{\text {identification }_{j}}$ ) during path delay testing. We further utilize these probability metrics for ranking the patterns on a sample circuit to illustrate the effectiveness of the proposed ranking method.

\section{CASE Study}

In this section, we illustrate our proposed delay probability metric by applying it on a sample circuit as shown in Fig. 2. For simplicity, we have considered a small circuit as a case study, but our metric can be applied to any large circuit. The sample circuit comprises interconnect models and gates connected to a global power supply voltage and ground networks. To study the impacts of PV and SN on path delay, we incorporate parameter variations in gates (at transistor level) and interconnects (on their widths) and then control the power supply and ground voltage locally (at gate level). The transistor and interconnect models are derived from 


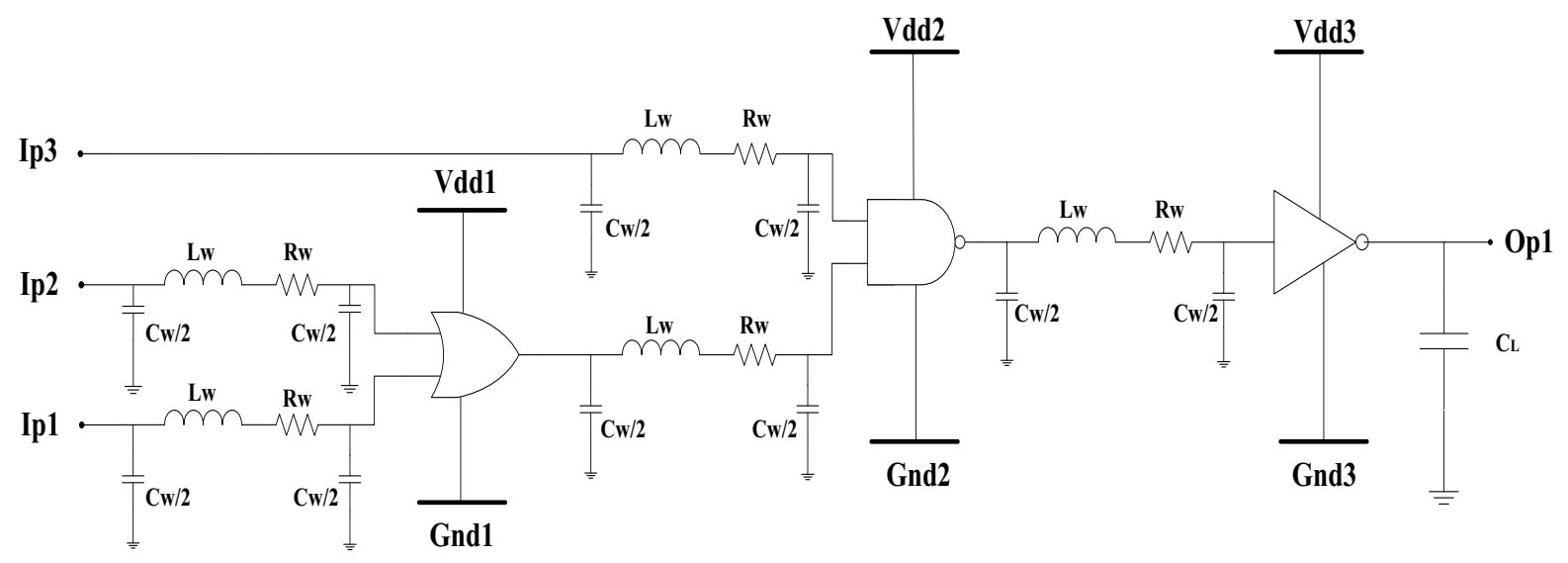

Fig. 2. SPICE circuit under the impact of process variations and supply noise

90nm Predictive Technology Model (PTM) [12]. HSPICE simulations are performed on the circuit for three different cases to analyze: (1) the impact of PV only, (2) the impact of SN only, and (3) the combined impact of PV and SN. For each case the following three steps are performed: (i) estimate path delay $\left(\delta_{\text {path }}\right)$, (ii) compute mean $\left(\mu_{P I_{j}}\right)$ and standard deviation $\left(\sigma_{P I_{j}}\right)$ from the delay probability distribution of each input pattern $\left(P I_{j}\right)$, and (iii) identify the worst-case path delay pattern $\left(P_{\text {identification }_{j}}\right)$ based on our ranking method. We utilize MATLAB to execute the mathematical computations of equations described in Section II.

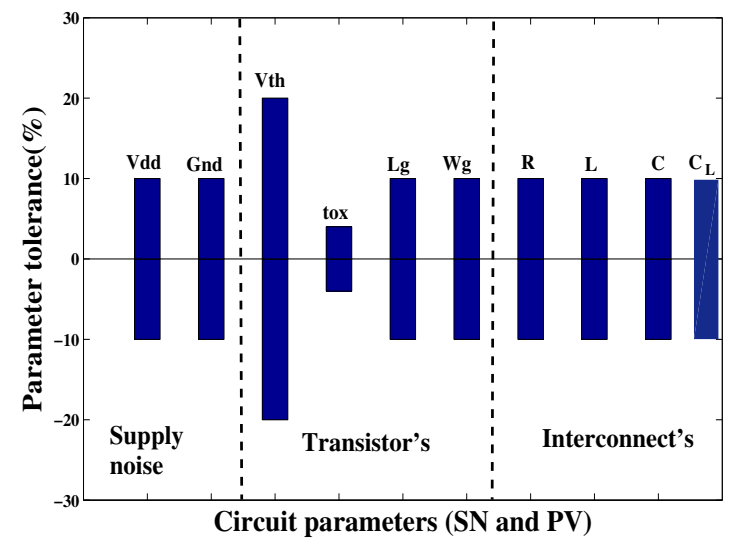

Fig. 3. Tolerance range of circuit parameters

Input vectors $\left(V_{1} V_{2}\right)$ are applied at each inputs $\{$ Ip1 Ip2 Ip3 $\}$ and their respective path delays are measured at $\{\mathrm{Op} 1\}$. Local supply voltage and input operating frequency are of $1 \mathrm{~V}$ and $1 \mathrm{GHz}$, respectively. We vary all the local supply voltages and the circuit parameters with their tolerance as shown in Fig. 3 [13]. Interconnects are modeled using $R L C \pi$-networks. Interconnect parameters $(R, L, C)$, transistor parameters $\left(V_{t h}\right.$, $\left.t_{o x}, L_{g}, W_{g}\right)$ and load capacitance $\left(C_{L}\right)$ are varied to model process variations. Local power supply voltages $\{$ Vdd1 Vdd 2
Vdd 3$\}$ and ground voltages $\{$ Gnd1 Gnd2 Gnd3 $\}$ are adapted to model supply noise at gate level. Path delay of the circuit can be measured between any two points, for our case study we observe between $\{\mathrm{Op} 1\}$ and $\{\mathrm{Ip} 1\}$.

We perform HSPICE simulations and measure the path delay for all the process corners in the circuit. Input pattern numbers, corresponding input vectors and their input transitions (i.e., rising and falling input signals) are shown in column I, column II and column III respectively of Table. I. Our delay probability metric can give all the possible path delays, but we are focused only on finding a worst-case path delay. Their corresponding metrics will indicate the input pattern to be the most effective for capturing path delay defects under PV and SN conditions.

\section{A. Case I: Impact of Process Variations}

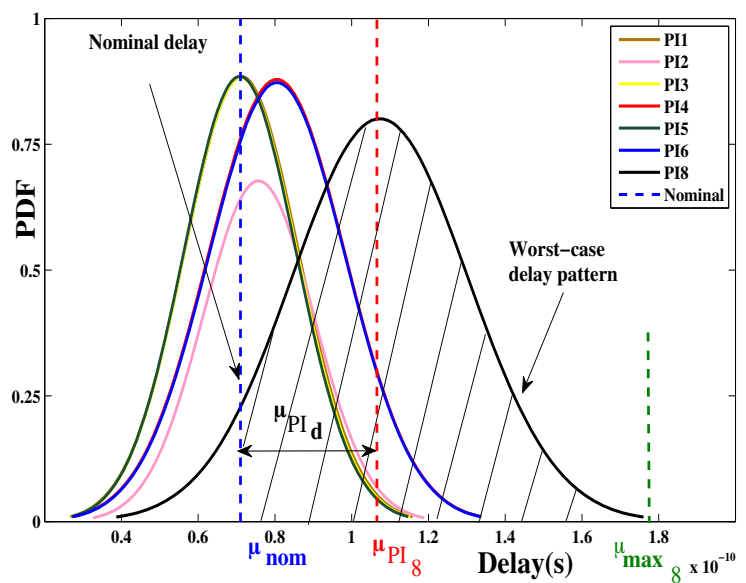

Fig. 4. Identification of worst-case path delay pattern under PV

In the first case, we study only the impact of PV, by varying the interconnect and transistor parameters while applying a nominal global supply voltage at their gates. Fig. 4 depicts the probability density distribution function of all the input 
TABLE I

RANKING METHOD FOR IDENTIFYING WORST-CASE PATH DELAY PATTERNS

\begin{tabular}{|c|c|c|c|c|c|c|c|c|c|c|c|}
\hline \multirow{2}{*}{ Pattern $\left(P I_{j}\right)$} & \multirow{2}{*}{ Input vectors $\left(V_{1} V_{2}\right)$ at $\{\operatorname{Ip} 1 \operatorname{Ip} 2 \operatorname{Ip} 3\}$} & \multirow{2}{*}{ Input transition } & \multicolumn{3}{|c|}{ Under PV } & \multicolumn{3}{|c|}{ Under SN } & \multicolumn{3}{|c|}{ Under PV and SN } \\
\hline & & & $\mu_{P I d}$ & $P_{i d n}$ & Rank & $\mu_{P I d}$ & $P_{i d n}$ & Rank & $\mu_{P I d}$ & $P_{i d n}$ & Rank \\
\hline PI1 & $\left\{\begin{array}{lll}10 & 10 & 10\end{array}\right\}$ & $\{$ Fall Fall Fall $\}$ & $0.27 \mathrm{ps}$ & 0.51 & 5 & 7.73ps & 0.67 & 5 & $1.12 \mathrm{ps}$ & 0.68 & 4 \\
\hline PI2 & $\left\{\begin{array}{lll}10 & 10 & 01\end{array}\right\}$ & $\{$ Fall Fall Rise $\}$ & $4.67 \mathrm{ps}$ & 0.60 & 4 & $7.97 \mathrm{ps}$ & 0.58 & 7 & $1.56 \mathrm{ps}$ & 0.63 & 7 \\
\hline PI3 & $\left\{\begin{array}{llll}10 & 01 & 10\end{array}\right\}$ & $\{$ Fall Rise Fall $\}$ & $0.07 \mathrm{ps}$ & 0.50 & 6 & $7.33 \mathrm{ps}$ & 0.66 & 4 & $1.22 \mathrm{ps}$ & 0.67 & 5 \\
\hline PI4 & $\left\{\begin{array}{llll}10 & 01 & 01\end{array}\right\}$ & $\{$ Fall Rise Rise $\}$ & $9.47 \mathrm{ps}$ & 0.70 & 3 & $17.6 \mathrm{ps}$ & 0.81 & 2 & $11.8 \mathrm{ps}$ & 0.89 & 2 \\
\hline PI5 & $\left\{\begin{array}{lll}01 & 10 & 10\end{array}\right\}$ & $\{$ Rise Fall Fall $\}$ & $0.13 \mathrm{ps}$ & 0.49 & 7 & $7.36 \mathrm{ps}$ & 0.67 & 6 & $1.5 \mathrm{ps}$ & 0.69 & 6 \\
\hline PI6 & $\left\{\begin{array}{lll}01 & 10 & 01\end{array}\right\}$ & $\{$ Rise Fall Rise $\}$ & $9.47 \mathrm{ps}$ & 0.70 & 2 & $17.3 \mathrm{ps}$ & 0.80 & 3 & $11.7 \mathrm{ps}$ & 0.89 & 3 \\
\hline PI7 & $\left\{\begin{array}{lll}01 & 01 & 10\end{array}\right\}$ & $\{$ Rise Rise Fall $\}$ & NA & NA & NA & NA & NA & NA & NA & NA & NA \\
\hline PI8 & $\left\{\begin{array}{lll}01 & 01 & 01\end{array}\right\}$ & $\{$ Rise Rise Rise $\}$ & $35.9 \mathrm{ps}$ & 0.94 & 1 & $48.2 \mathrm{ps}$ & 0.98 & 1 & $25.9 \mathrm{ps}$ & 0.99 & 1 \\
\hline
\end{tabular}

$* \mu_{P I d}=$ Difference between nominal delay $\left(\mu_{n o m}\right)$ and delay mean of an input pattern $\mu(P i)$

$* P_{i d n}=P_{\text {identification }}$ i.e., exposed area of the probability density function. $*$ NA $=$ No output transition at launch cycle, no no delay measured

patterns under PV. For each input pattern, their respective $\mu_{P I d}, P_{\text {identification }}$ and rank are listed in column IV, V and VI of Table. I. Using our probabilistic pattern ranking method, we obtain $\mathrm{PI}_{8}$ as the worst-case path delay pattern under the impact of PV. This is also shown in Fig. 4 as the pattern with the largest area exposed beyond the nominal delay threshold line.

\section{B. Case II: Impact of Supply Noise}

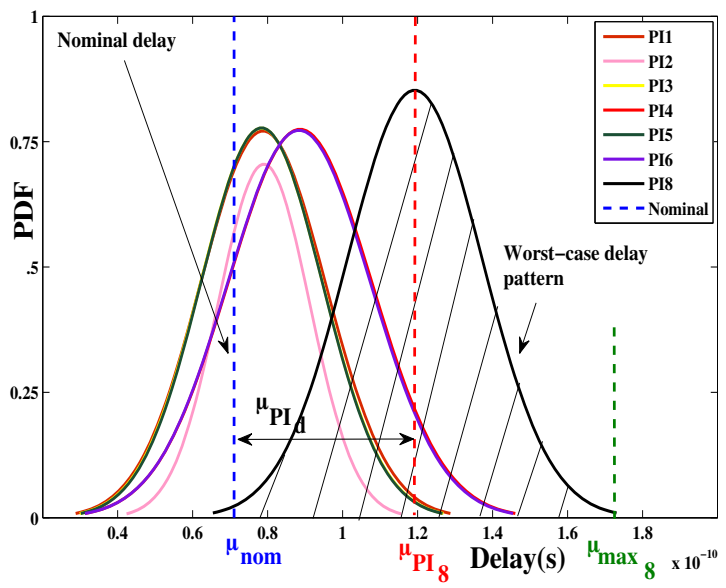

Fig. 5. Identification of worst-case path delay pattern under SN

In this case, we study only the impact of SN, by locally varying power supply and ground voltage, while considering no process variation on transistors and interconnects. Fig. 5 depicts the probability density distribution function of all the input patterns under SN. For each input pattern, their respective $\mu_{P I d}, P_{\text {identification }}$ and rank are listed in column VII, VIII and IX of Table. I. Using our probabilistic pattern ranking method, we obtain $P I_{8}$ as the worst-case path delay pattern under the impact of SN. After comparing Fig. 4 and Fig. 5, the changes in the delay distribution for the same input pattern can be noticed; indicating the higher impact of SN than PV.

\section{Case III: Impact of Process Variations and Supply Noise}

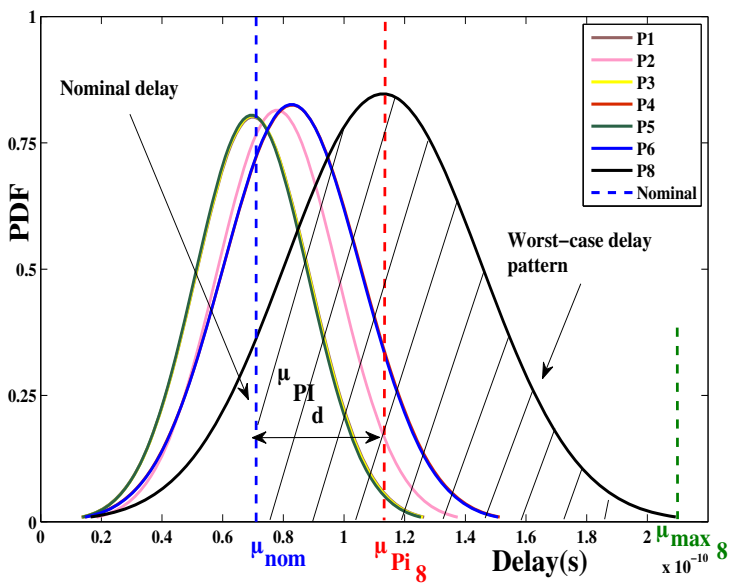

Fig. 6. Identification of worst-case delay pattern under PV and SN

In the third case, we investigate the combined impact of PV and SN. Fig. 6 depicts the probability density distribution function of all the input patterns under PV and SN. For each input pattern, their respective $\mu_{P I d}, P_{\text {identification }}$ and rank are listed in column X, XI and XII of Table. I. Based on our probabilistic pattern ranking method, we obtain $P I_{8}$ as the worst-case path delay pattern under the combined impact of PV and SN. Please note that, while $P I_{8}$ pattern was also identified in case I and II, the value of the probability density function for path delay varies.

The results of these case studies indicate that by applying the proposed ranking method, we can identify the pattern(s) that lead to the worst-case path delay when PV and SN conditions are present.

\section{EXPERIMENTAL RESUltS}

In this section, we present our experimental results based on five ITC'99 benchmark circuits [14]. We apply our probabilistic method on a single critical path, however it can be 
TABLE II

RESULTS OF ITC'99 BENCHMARK CIRCUITS

\begin{tabular}{|c|c|c|c|c|c|c|c|c|c|c|}
\hline \multirow{2}{*}{ Ckt } & \multirow{2}{*}{$\mathrm{X}$-bit input pattern } & \multicolumn{4}{|c|}{ ATPG } & \multicolumn{4}{|c|}{ Our method } & \multirow[b]{2}{*}{$\% \mu_{P I d}$} \\
\hline & & Input pattern & $\mu_{P I d}$ & $P_{i d n}$ & Rank & Input pattern & $\mu_{P I d}$ & $P_{i d n}$ & Rank & \\
\hline b01 & $11001 X$ & 110010 & $136 \mathrm{ps}$ & 0.91 & 2 & 110011 & $140.77 \mathrm{ps}$ & 0.95 & 1 & $3.3 \%$ \\
\hline b02 & $0000 \boldsymbol{X}$ & 00000 & $159.34 \mathrm{ps}$ & 0.96 & 2 & 00001 & $167 \mathrm{ps}$ & 0.93 & 1 & $4.5 \%$ \\
\hline b05 & $00 X 0 X$ & 00100 & $171.9 \mathrm{ps}$ & 0.84 & 4 & 00001 & 191.3ps & 0.91 & 1 & $10.14 \%$ \\
\hline b06 & $100 X 1 X$ & 100110 & $123 \mathrm{ps}$ & 0.93 & 3 & 100011 & $147.5 \mathrm{ps}$ & 0.89 & 1 & $16.6 \%$ \\
\hline b11 & $X X X X X X X 000 X$ & 11000110001 & $172.76 \mathrm{ps}$ & 0.82 & 7 & $01 \times X X X X 0000$ & $202.42 \mathrm{ps}$ & 0.98 & 1 & $14.65 \%$ \\
\hline
\end{tabular}

applied to any paths. Table. II shows the summary of our experimental results. We utilized an ATPG tool for generating X-bit input patterns mentioned in column II. Then, we filled only the relevant X-bits (indicated in italics and bold letters) based on efficient X-filling method [15].

We explain in detail the results of our delay probability metric for b05 benchmark circuit. For all the X-filled input patterns (i.e., 00000, 00001, 00100, and 00101), we computed the mean delay difference $\mu_{P I d}$ (i.e., $176.9 \mathrm{ps}, 191.3 \mathrm{ps}, 171.9 \mathrm{ps}$, $182.67 \mathrm{ps}$ ) and $P_{\text {identification }}$ (i.e., $0.86,0.91,0.84,0.90$ ), and then ranked (i.e., 3, 1, 4 and 2) each input pattern. We selected the input pattern with rank 1 (i.e., 00001 as shown in column VII), as by our method this pattern has the highest probability to give the worst-case path delay under the impact of PV and SN. Also, we selected the input pattern generated by the ATPG tool (i.e., 00100 as shown in column III) and indicate its rank in column VI. In column XI, the mean delay difference (i.e., $10.14 \%$ ) between the two patterns (our method pattern and ATPG pattern) is obtained. Such discrepancies further indicate the need for investigate the worst-case path delay problem and reveal the effectiveness of our method in ranking and selecting input patterns that take into account process variation and supply noise issues.

The pattern generated by random X-filling using the ATPG tool differs from the pattern generated by our probabilistic method. This indicates that, while a test pattern sensitizes a path for path delay testing, it doesn't necessarily capture its worst-case path delay. Whereas, proposed method, investigates a set of patterns and aims to rank them based on the likeliness to obtain the worst path delay when process variation and supply noise variations are taken into account. The proposed method is practical to be embedded on pattern generation flow i.e., post-ATPG X-filling, which is also the focus of our future work.

\section{CONCLUSIONS}

In this paper, we proposed a delay probability metric for identifying a worst-case path delay pattern under the impact of process variation and supply noise. The presented probabilistic pattern ranking method aims at capturing delay defects during path delay test. Our experimental results on ITC'99 benchmark circuits suggests to improve the existing pattern generation methods by incorporating the impacts of PV and SN. As future research, we aim to implement the probabilistic method in $\mathrm{X}$ filling pattern generation flow.

\section{REFERENCES}

[1] B. Li, N. Chen, M. Schmidt, W. Schneider, and U. Schlichtmann, "On hierarchical statistical static timing analysis," in Design, Automation Test in Europe Conference Exhibition, 2009. DATE '09., 2009, pp. 13201325.

[2] F. Galarza-Medina, J. Garcia-Gervacio, V. Champac, and A. Orailoglu, "Small-delay defects detection under process variation using Inter-Path Correlation," in VLSI Test Symposium (VTS), 2012 IEEE 30th, 2012, pp. $127-132$.

[3] X. Wang, M. Tehranipoor, and R. Datta, "Path-RO: A novel on-chip critical path delay measurement under process variations," in ComputerAided Design, 2008. ICCAD 2008. IEEE/ACM International Conference on, 2008, pp. 640-646.

[4] R. Tayade, S. Sundereswaran, and J. Abraham, "Small-Delay Defect Detection in the Presence of Process Variations," in Quality Electronic Design, 2007. ISQED '07. 8th International Symposium on, 2007, pp. 711-716.

[5] G. Yu, W. Dong, Z. Feng, and P. Li, "A Framework for Accounting for Process Model Uncertainty in Statistical Static Timing Analysis," in Design Automation Conference, 2007. DAC '07. 44th ACM/IEEE, 2007, pp. 829-834.

[6] A. Todri, A. Bosio, L. Dilillo, P. Girard, and A. Virazel, "Uncorrelated Power Supply Noise and Ground Bounce Consideration for Test Pattern Generation," in IEEE Transactions on VLSI Systems, 2013, pp. 958-970.

[7] H. Xu, V. Pavlidis, W. Burleson, and G. De Micheli, "The combined effect of process variations and power supply noise on clock skew and jitter," in Quality Electronic Design (ISQED), 2012 13th International Symposium on, 2012, pp. 320-327.

[8] K. Peng, M. Yilmaz, M. Tehranipoor, and K. Chakrabarty, "Highquality pattern selection for screening small-delay defects considering process variations and crosstalk," in Design, Automation Test in Europe Conference Exhibition (DATE), 2010, 2010, pp. 1426-1431.

[9] M. Orshansky and K. Keutzer, "A general probabilistic framework for worst case timing analysis," in Design Automation Conference, 2002. Proceedings. 39th, 2002, pp. 556-561.

[10] N. Weste and K. Eshraghian, "Principles of cmos vlsi design: A systems perspective," in 2nd Edition. Addison - Wesley, New York, 1993.

[11] Y. Ismail, E. Friedman, and J. Neves, "Equivalent Elmore delay for RLC trees," in Design Automation Conference, 1999. Proceedings. 36th, 1999, pp. 715-720.

[12] Predictive Technology Model (PTM). [Online]. Available: http://ptm.asu.edu/

[13] X. Qi, S. Lo, Y. Luo, A. Gyure, M. Shahram, and K. Singhal, "Simulation and analysis of inductive impact on VLSI interconnects in the presence of process variations," in Custom Integrated Circuits Conference, 2005. Proceedings of the IEEE 2005, 2005, pp. 309-312.

[14] ITC'99 Benchmark circuits (2003). [Online]. Available: http://www.cad.polito.it/downloads/tools/itc99.html

[15] A. Asokan, A. Todri-Sanial, A. Bosio, L. Dilillo, P. Girard, S. Pravossoudovitch, and A. Virazel, "Path Delay Test in the Presence of MultiAggressor Crosstalk, Power Supply Noise and Ground Bounce," in Design and Diagnostics of Electronic Circuits and Systems,. 17th, 2014. 